\title{
Extensive aortic valve ring abscess formation: a rare complication of $\mathrm{Q}$ fever endocarditis
}

\author{
S. Fort, A.G. Fraser and K.A.A. Fox \\ Department of Cardiology, University Hospital of Wales, Heath Park, Cardiff CF4 4XW, UK.
}

\begin{abstract}
Summary: We report the successful management and 2 year follow up of a young patient with $Q$ fever endocarditis on a congenitally bicuspid aortic valve complicated by extensive abscess formation in the aortic valve ring and interventricular septum. Aortic root abscess formation complicating $\mathbf{Q}$ fever endocarditis has been reported in only one previous patient. Serological tests may thus be indicated in patients with aortic abscesses.

Despite extensive aortic and intramyocardial abscess formation it proved possible to control the progression of disease by open drainage of the abscess and aortic valve replacement. Although the requirement for aortic root replacement was anticipated in this patient, it has not been required.
\end{abstract}

\section{Introduction}

$\mathrm{Q}$ fever is a systemic zoonosis caused by Coxiella burnetii. Endocarditis is one of the most important of its many clinical manifestations, and predominantly affects the aortic valve. Prolonged antibiotic treatment remains the first line therapy of $Q$ fever endocarditis, with surgery reserved for severe haemodynamic complications. We report a case of $Q$ fever endocarditis complicated by extensive suppuration affecting the aortic valve ring and myocardium.

\section{Case report}

A 21 year old male presented with a 2-week history of lethargy, perspiration, rigors, night sweats, weight loss, palpitations and breathlessness. Twelve months previously his general practitioner had detected a heart murmur and hypertension $(160 / 110 \mathrm{mmHg})$. Coarctation was considered because of reduced femoral pulses, but aortography demonstrated a bicuspid aortic valve and pseudo-coarctation. There was no evidence of infective endocarditis and the aortic root was normal.

Five months prior to the presentation with endocarditis, the patient suffered a 'flu-like' illness with fever, rigors, pleuritic chest pain and haemoptysis. The chest radiograph demonstrated left basal consolidation, but the causative organism was never identified.

On presentation to the cardiac unit he was pyrexial $\left(39^{\circ} \mathrm{C}\right)$ and he had finger clubbing, a single splinter haemorrhage and two Janeway lesions. His blood

Correspondence: S. Fort, M.R.C.P.

Accepted: 1 December 1988. pressure was $140 / 70 \mathrm{mmHg}$. He had a loud ejectio systolic murmur, as before, but in addition a new and. long early diastolic murmur of aortic regurgitation. $\subseteq$

A chest radiograph demonstrated a normal hear size, with no evidence of pulmonary oedema. An electrocardiogram demonstrated first degree hemin block (PR interval 0.26 seconds) and right bundle branch block. The serum haemoglobin was 11.5 dif with a white cell count of $10.3 \times 10^{9} / 1$, and the bloos film demonstrated early iron deficiency changes. Tho erythrocyte sedimentation rate was $38 \mathrm{~mm} / \mathrm{h}$. Six set⿱艹 of blood cultures, a mid-stream specimen of urine, an $\$$ nasal and throat swabs were all negative. There was $n \overrightarrow{8}$ microscopic haematuria. Immunoglobulin $\mathbf{G}$ level@ were elevated to $24.1 \mathrm{~g} / \mathrm{l}$.

On a presumptive diagnosis of infective endocard itis he was treated with high doses of benzyl penicilling flucloxacillin and gentamicin intravenously, but he remained febrile. Antimicrobial treatment was changed to tetracycline and co-trimoxazole based upon the results of serology. The Phase I and Phase I\$ Coxiella burnetii titres were elevated to dilutions ox $1: 1024$ and $1: 8192$ respectively indicating the diag? nosis of $\mathrm{Q}$ fever. Coxiella burnetii was subsequentl isolated from guinea pigs inoculated with materias. from the excised aortic valve. On detailed enquiry the patient admitted to a single episode of exposure to farm animals.

Echocardiography demonstrated a calcified bicusస్త pid aortic valve with a large vegetation on the right (OD anterior) coronary cusp measuring $1.5 \times 2.2 \mathrm{~cm}$ There was an adjacent abscess formation in the aorti $\xi_{0}$ root which extended into the interventricular septunf? (see Figures 1 and 2). Doppler echocardiography dido 


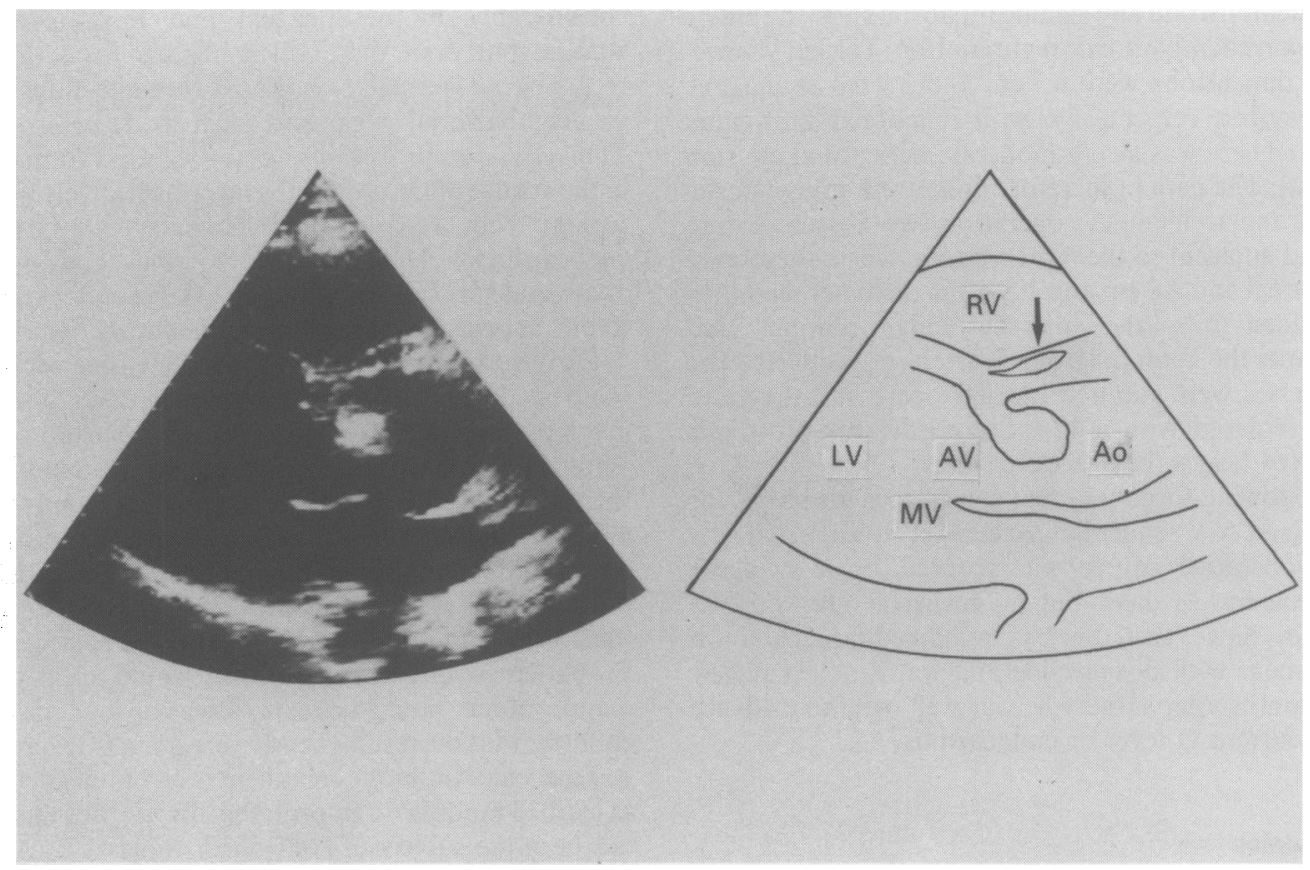

Figure 1 Parasternal long axis echocardiogram demonstrating abscess in anterior aortic root (arrow) which extends into the upper interventricular septum, and a large vegetation on the right coronary cusp of the aortic valve.

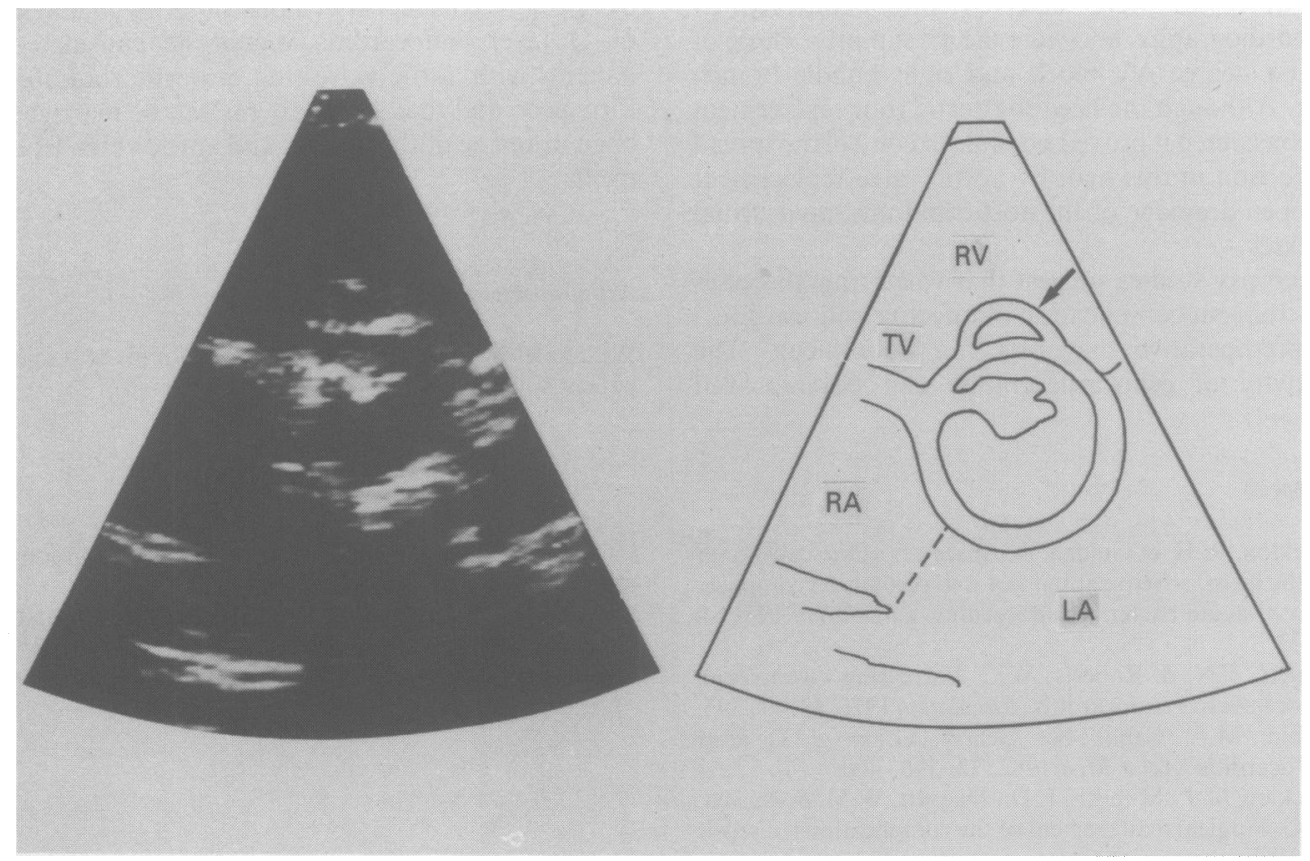

Figure 2 Short axis echocardiogram through the aortic root. An abscess is seen anteriorly (arrow), bulging into the right ventricular outflow tract below the pulmonary valve. 
not demonstrate any significant aortic stenosis, but he had considerable aortic regurgitation. The left ventricular dimensions were $6.7 \mathrm{~cm}$ and $3.9 \mathrm{~cm}$ in diastole and systole respectively, with functional shortening $42 \%$. There was also a moderate pericardial effusion $(1 \mathrm{~cm})$. The patient therefore underwent urgent aortic valve replacement. At operation two abscesses were found adjacent to the right coronary cusp; these were spherical and $2.0 \mathrm{~cm}$ and $1.5 \mathrm{~cm}$ in diameter, and they extended into the interventricular septum, and towards the main pulmonary artery respectively. The abscesses were curetted but left open, and the valve was replaced with a 13A Starr Edwards prosthesis inserted below the abscess cavities.

Following surgery he had complete heart block, for which an A-V sequential pacemaker was inserted. His post-operative course was marked by a swinging pyrexia for 18 days, but no focus of infection was found. Since then he has remained apyrexial on treatment with doxycycline and warfarin. At review 24 months after surgery he was well with no evidence of recurrent $Q$ fever or endocarditis.

\section{Discussion}

This patient is unusual because he had aortic valve ring abscess formation, with intra-myocardial extension and heart block, complicating $\mathrm{Q}$ fever endocarditis. The abscesses were detected pre-operatively by echocardiography, and were the presumptive cause of his first degree AV block and right bundle branch block. Although the need for aortic root replacement was considered it proved possible to control the spread of infection in this man by aortic valve replacement and open drainage of the aortic and intramyocardial abscesses.

Necropsy studies suggest that valve ring abscesses are a frequent complication of infective endocarditis, ${ }^{1}$ but pre-operative diagnosis can be difficult. The sensitivity of echocardiography can be improved

\section{References}

1. Sheldon, W.H. \& Golden, A. Abscesses of the valve rings of the heart, a frequent but not well recognised complication of acute bacterial endocarditis. Circulation 1951, 4: $1-12$.

2. Arnett, E.N. \& Roberts, W.C. Valve ring abscesses in acute infective endocarditis. Circulation 1976, 1: 140-145.

3. Tobin, M.J., Cahill, N., Gearty, G. et al. Q fever endocarditis. Am J Med 1982, 72: 396-400.

4. Buckley, M.J., Mundth, E.D., Daggett, W.M. \& Austen, W.G. Surgical management of the complications of sepsis involving the aortic valve, aortic root and ascending aorta. Ann Thorac Surg 1971, 12: 391-399. considerably by oesophageal and intra-operative studies, but even then, when abscess formation recognized it is usually associated with infections with virulent bacterial organisms such as Streptococcu $\frac{\mathbb{B}}{8}$ Staphylococcus or Pneumococcus. ${ }^{2}$ Abscess formation. in the aortic root was detected intra-operatively in one patient from a series of 10 patients with $Q$ feveff endocarditis. ${ }^{3}$ However, valve ring and intra? myocardial abscess formation in $\mathbf{Q}$ fever endocarditis to our knowledge, has not been previously described

Early surgical intervention with extensive excisio $\$$ of infected material is recommended in cases of sepsiक involving the aortic valve ring and aortic root. Without this, and in spite of appropriate antibiotic therapy, mortality is high because of continuing infection, aortic regurgitation and heart failure. Since it may be difficult to control infection in $\mathrm{Q}$ fever and to prevent subsequent prosthetic valve endocarditiș. there is a temptation to postpone valve replacemen In patients with abscesses this would clearly bi inappropriate, and the experience of our patient confirms that the result of early surgery in $Q$ fever ma $x_{0}$ be excellent. Antibiotic treatment is recommended for at least 12 months $^{5,6}$ and probably for life, but this has not been the subject of controlled studies.

The clinical features of $\mathbf{Q}$ fever are protean, bue endocarditis is the most serious complication. This can occur between one and 20 years after the acyte infection, and it is associated with a mortalityo $31-56 \% .{ }^{5,6}$ This report demonstrates that a diagnosis of $Q$ fever endocarditis should be considered patients with aortic valve ring or aortic root abscess formation and that such patients can be managed b open drainage of the abscess and aortic valve replace? ment.

\section{Acknowledgement}

We thank Mr S.H. Armistead, F.R.C.S., for his help with th patient's management.

5. Turck, W.P.G., Howitt, G., Turnberg, L.A. et al. Chronif $Q$ fever. $Q J$ Med 1976, 45: $193-217$.

6. Wilson, H.G., Neilson, G.H., Galea, E.G., Stafford, G. O'Brien, M.F. Q fever endocarditis in Queensland. $\mathrm{Cir}_{\overline{\mathrm{N}}}$ culation 1976, 53: 680-684. 